\title{
On the dichromatic number of surfaces
}

\author{
Pierre Aboulker \\ DIENS, École Normale Supérieure, CNRS \\ PSL University, Paris, France \\ pierreaboulker@gmail.com
}

\author{
Frédéric Havet \\ CNRS, Université Côte d'Azur, I3S, INRIA \\ Sophia Antipolis, France \\ frederic.havet@i3s.unice.fr
}

\author{
Kolja Knauer \\ Aix Marseille Univ, Université de Toulon, CNRS, LIS \\ Marseille, France \\ Departament de Matemàtiques i Informàtica \\ Universitat de Barcelona, Spain \\ kolja.knauer@ub.edu \\ Clément Rambaud \\ DIENS, École Normale Supérieure, CNRS \\ PSL University, Paris, France \\ clement.rambaud@ens.psl.eu
}

Submitted: Feb 4, 2021; Accepted: Jan 31, 2022; Published: Feb 11, 2022

(C) The authors. Released under the CC BY-ND license (International 4.0).

\begin{abstract}
In this paper, we give bounds on the dichromatic number $\vec{\chi}(\Sigma)$ of a surface $\Sigma$, which is the maximum dichromatic number of an oriented graph embeddable on $\Sigma$. We determine the asymptotic behaviour of $\vec{\chi}(\Sigma)$ by showing that there exist constants $a_{1}$ and $a_{2}$ such that, $a_{1} \frac{\sqrt{-c}}{\log (-c)} \leqslant \vec{\chi}(\Sigma) \leqslant a_{2} \frac{\sqrt{-c}}{\log (-c)}$ for every surface $\Sigma$ with Euler characteristic $c \leqslant-2$. We then give more explicit bounds for some surfaces with high Euler characteristic. In particular, we show that the dichromatic numbers of the projective plane $\mathbb{N}_{1}$, the Klein bottle $\mathbb{N}_{2}$, the torus $\mathbb{S}_{1}$, and Dyck's surface $\mathbb{N}_{3}$ are all equal to 3 , and that the dichromatic numbers of the 5 -torus $\mathbb{S}_{5}$ and the 10 cross surface $\mathbb{N}_{10}$ are equal to 4 . We also consider the complexity of deciding whether a given digraph or oriented graph embeddable on a fixed surface is $k$-dicolourable. In particular, we show that for any fixed surface, deciding whether a digraph embeddable on this surface is 2-dicolourable is NP-complete, and that deciding whether a planar oriented graph is 2-dicolourable is NP-complete unless all planar oriented graphs are 2-dicolourable (which was conjectured by Neumann-Lara).
\end{abstract}

Mathematics Subject Classifications: 05C15,05C20,05C10 


\section{Introduction}

All surfaces considered in this paper are closed.

A graph is embeddable on a surface $\Sigma$ if its vertices can be mapped onto distinct points of $\Sigma$ and its edges onto simple curves of $\Sigma$ joining the points onto which its endvertices are mapped, so that two edge curves do not intersect except in their common extremity. A face of an embedding $\tilde{G}$ of a graph $G$ is a component of $\Sigma \backslash \tilde{G}$. Recall that an important theorem of the topology of surfaces, known as the Classification Theorem for Surfaces, states that every surface is homeomorphic to either the $k$-torus - a sphere with $k$-handles $\mathbb{S}_{k}$ or the $k$-cross surface - a sphere with $k$-cross-caps $\mathbb{N}_{k}$. The surface $\mathbb{S}_{0}=\mathbb{N}_{0}$ is the sphere, and the surfaces $\mathbb{S}_{1}, \mathbb{S}_{2}, \mathbb{N}_{1}, \mathbb{N}_{2}, \mathbb{N}_{3}$ are also called the torus, the double torus, the projective plane, the Klein bottle, and Dyck's surface, respectively. The Euler characteristic of a surface homeomorphic to $\mathbb{S}_{k}$ is $2-2 k$ and of a surface homeomorphic to $\mathbb{N}_{k}$ it is $2-k$. We denote the Euler characteristic of a surface $\Sigma$ by $c(\Sigma)$.

Let $G$ be a graph. We denote by $n(G)$ its number of vertices, and by $m(G)$ its number of edges. If $G$ is embedded in a surface $\Sigma$, then we denote by $f(G)$ the number of faces of the embedding. Euler's Formula relates the numbers of vertices, edges and faces of a (connected) graph embedded in a surface.

Theorem 1. EuleR's Formula

Let $G$ be a connected graph embedded on a surface $\Sigma$. Then

$$
n(G)-m(G)+f(G) \geqslant c(\Sigma) .
$$

We denote by $\operatorname{Ad}(G)=2 m / n$ the average degree of a graph $G$. Euler's formula implies that graphs on surfaces have bounded average degree.

Theorem 2. A graph $G$ embeddable on a surface $\Sigma$ satisfies:

$$
m(G) \leqslant 3 n(G)-3 c(\Sigma) \quad \text { and } \quad \operatorname{Ad}(G) \leqslant 6-\frac{6 c(\Sigma)}{n(G)} .
$$

Moreover, there is equality if and only if $G$ is a triangulation.

A $\boldsymbol{k}$-colouring of a graph $G$ is a partition of the vertex set of $G$ into $k$ disjoint stable sets (i.e. sets of pairwise non-adjacent vertices). A graph is $\boldsymbol{k}$-colourable if it has a $k$-colouring. The chromatic number of a graph $G$, denoted by $\chi(G)$, is the least integer $k$ such that $G$ is $k$-colourable, and the chromatic number of a surface $\Sigma$, denoted by $\chi(\Sigma)$, is the least integer $k$ such that every graph embeddable on $\Sigma$ is $k$-colourable. Determining the chromatic number of surfaces attracted lots of attention, with its most important instance being the Four Colour Conjecture on planar graphs (i.e. graphs embeddable on $\mathbb{S}_{0}$ ). This conjecture was eventually proved by Appel and Haken [2] using computer assistance and another proof requiring less computer assistance was given by Robertson et al. [32]. Maybe surprisingly, the chromatic numbers of surfaces other than the plane were established before the Four Colour Theorem. In 1890, Heawood [19] proved the following theorem as a consequence of Euler's Formula. 
Theorem 3 (Heawood [19]). If $\Sigma$ is a surface with Euler characteristic $c \leqslant 0$, then $\chi(\Sigma) \leqslant H(c)=\left\lfloor\frac{7+\sqrt{49-24 c}}{2}\right\rfloor$.

Franklin [12] showed that the above inequality is not tight as the Klein bottle has chromatic number 6 (the above inequality yields 7 as the Klein bottle has Euler characteristic 0). Contrary to the sphere, on other surfaces the most effort of determining the chromatic number went into proving the lower bounds. Indeed, Ringel and Youngs [31] proved that the Klein bottle is the sole surface that does not admit an embedding of a complete graph witnessing the Heawood bound.

Theorem 4 (Ringel and Youngs [31]). Let $\Sigma$ be a surface different from the Klein bottle $\mathbb{N}_{2}$ and let $c$ be its Euler characteristic. Then the complete graph of order $H(c)$ is embeddable on $\Sigma$.

The girth of a graph is the length of a shortest cycle in it (or $+\infty$ if it is acyclic). The chromatic number of graphs embeddable on a surface and of girth at least $g$ has been studied. In the same way as Theorem 2, one can derive from Euler's Formula that the average degree of a graph $G$ embeddable on a surface $\Sigma$ and of girth at least $g$ is at most $\frac{2 g}{g-2}-\frac{2 g c(\Sigma)}{n(G)}$. This implies that the maximum chromatic number over all graphs of girth at least $g$ embeddable on $\Sigma$ tends to 3 when $g$ tends to $+\infty$. A particular interest has been devoted to triangle-free graphs, i.e., graphs of girth at least 4 . The above bound on the average degree implies that triangle-free planar graphs have average degree at most 3 , and so are 4-colourable. The celebrated Grötzsch's Theorem [17] asserts that such graphs are even 3-colourable. A short proof can be found in [35].

Theorem 5 (Grötzsch [17]). Every triangle-free planar graph is 3-colourable.

Kronk and White [24] proved that every triangle-free graph embeddable on the torus is 4-colourable, and Kronk [23] studied the chromatic number of triangle-free graphs on certain surfaces. Asymptotic bounds on the maximum chromatic number of triangle-free graphs embeddable on a given surface have been given by Gimbel and Thomassen [16]. Here we only give the results for orientable surfaces.

Theorem 6 (Gimbel and Thomassen [16]). There exist positive constants $c_{1}$ and $c_{2}$ such that the following hold:

(i) Every triangle-free graph embeddable on $\mathbb{S}_{k}$ has chromatic number at most $c_{1} \sqrt[3]{\frac{k}{\log k}}$.

(ii) for each $k$, there exists a triangle-free graph which is embeddable on $\mathbb{S}_{k}$ and with chromatic number at least $c_{2} \frac{\sqrt[3]{k}}{\log k}$.

In 1982, Neumann Lara [28] introduced the notion of directed colouring or dicolouring. A $\boldsymbol{k}$-dicolouring of a digraph is a partition of its vertex set into $k$ subsets inducing acyclic subdigraphs. A digraph is $\boldsymbol{k}$-dicolourable if it has a $k$-dicolouring. The dichromatic 
number of a digraph $D$, denoted by $\vec{\chi}(D)$, is the least integer $k$ such that $D$ is $k$ dicolourable.

Let $G$ be an undirected graph. The bidirected graph $\overleftrightarrow{G}$ is the digraph obtained from $G$ by replacing each edge by a digon, that is a pair of oppositely directed arcs between the same end-vertices. Observe that $\chi(G)=\vec{\chi}(\overleftrightarrow{G})$ since any two adjacent vertices in $\overleftrightarrow{G}$ induce a directed cycle of length 2 .

The digirth of a digraph is the length of a smallest directed cycle in it (or $+\infty$ if it is acyclic). In view of the influence of the girth on the chromatic number of graphs on surfaces, it is natural to study the influence of the digirth on the dichromatic number. In particular, it is interesting to study the dichromatic number of digraphs of digirth 3 , which are called oriented graphs. Alternatively, oriented graphs may be seen as the digraphs which can be obtained from (simple) graphs by orienting every edge, that is replacing each edge by exactly one of the two possible arcs between its end-vertices. If $\vec{G}$ is obtained from $G$ by orienting its edges, we say that $G$ is the underlying graph of $\vec{G}$. It is easy to show that oriented planar graphs are 3-dicolourable and Neumann Lara [28] proposed the following conjecture, which can be viewed as an analogue of Grötzsch's Theorem (Theorem 5).

Conjecture 7 (Neumann Lara [28]). Every oriented planar graph is 2-dicolourable.

The conjecture is part of an active field of research. It has been verified for planar oriented graphs on at most 26 vertices [20] and holds for planar digraphs of digirth at least 4 [25].

Analogously to the history of the chromatic number, in the present paper we study the dichromatic number of surfaces. The dichromatic number of a surface $\Sigma$, denoted by $\vec{\chi}(\Sigma)$, is the least integer $k$ such that every oriented graph embeddable on $\Sigma$ is $k$ dicolourable.

The arboricity of a graph $G$, denoted by $a(G)$, is the minimum integer $k$ such that $V(G)$ can be partitioned into $k$ sets, each of which induces a forest, that is an acyclic (non-directed) graph.

Let $\vec{G}$ be an oriented graph and $G$ its underlying graph. Then

$$
\vec{\chi}(\vec{G}) \leqslant a(G) \leqslant \chi(G)
$$

Kronk [22] proved that if a graph is embeddable on a surface $\Sigma$ with Euler characteristic $c \leqslant 1$, then $a(G) \leqslant\left\lfloor\frac{9+\sqrt{49-24 c(\Sigma)}}{4}\right\rfloor$. By Eq. (1), this immediately implies

$$
\vec{\chi}(\Sigma) \leqslant\left\lfloor\frac{9+\sqrt{49-24 c(\Sigma)}}{4}\right\rfloor
$$

We first improve on this bound by determining the asymptotic behaviour of $\vec{\chi}(\Sigma)$. We show in Theorem 21, that there exists two constants $a_{1}$ and $a_{2}$ such that, for every surface $\Sigma$ with Euler characteristic $c \leqslant-2$, we have

$$
a_{1} \frac{\sqrt{-c}}{\log (-c)} \leqslant \vec{\chi}(\Sigma) \leqslant a_{2} \frac{\sqrt{-c}}{\log (-c)} .
$$


We then estimate the exact value of the dichromatic number of surfaces close to the sphere. Table 1 summarizes the main results.

\begin{tabular}{|c|c|c|c|}
\hline$\Sigma$ & $c(\Sigma)$ & Bounds for $\vec{\chi}(\Sigma)$ & Reference \\
\hline \hline Sphere $\mathbb{N}_{0}=\mathbb{S}_{0}$ & 2 & $2 \leqslant \vec{\chi} \leqslant 3$ & Neumann Lara [28] \\
\hline Projective plane $\mathbb{N}_{1}$ & 1 & $\vec{\chi}=3$ & Corollary 24 \\
\hline Klein bottle $\mathbb{N}_{2}$ & 0 & $\vec{\chi}=3$ & Corollary 24 \\
\hline Torus $\mathbb{S}_{1}$ & 0 & $\vec{\chi}=3$ & Corollary 24 \\
\hline Dyck's surface $\mathbb{N}_{3}$ & -1 & $\vec{\chi}=3$ & Corollary 24 \\
\hline $\mathbb{S}_{2}, \mathbb{N}_{4}$ & -2 & $3 \leqslant \vec{\chi} \leqslant 4$ & Theorems 22 and 27 \\
\hline $\mathbb{N}_{5}$ & -3 & $3 \leqslant \vec{\chi} \leqslant 4$ & Theorems 22 and 27 \\
\hline $\mathbb{S}_{3}, \mathbb{N}_{6}$ & -4 & $3 \leqslant \vec{\chi} \leqslant 4$ & Theorems 22 and 27 \\
\hline $\mathbb{N}_{7}$ & -5 & $3 \leqslant \vec{\chi} \leqslant 4$ & Theorems 22 and 27 \\
\hline $\mathbb{S}_{4}, \mathbb{N}_{8}$ & -6 & $3 \leqslant \vec{\chi} \leqslant 4$ & Theorems 22 and 27 \\
\hline $\mathbb{N}_{9}$ & -7 & $3 \leqslant \vec{\chi} \leqslant 4$ & Theorems 22 and 27 \\
\hline $\mathbb{S}_{5}, \mathbb{N}_{10}$ & -8 & $\vec{\chi}=4$ & Corollary 28 \\
\hline
\end{tabular}

Table 1: Bounds on the dichromatic number of some surfaces.

Finally, we consider the computational complexity of the related (di)colourability problems. Regarding undirected graphs, for any surface $\Sigma$ and any integer $k \geqslant 5$, there are only finitely many $(k+1)$-critical graphs (i.e. graphs $G$ such that $\chi(G)=k+1$ and $\chi(H) \leqslant k$ for any proper subgraph $H$ of $G$ ) embeddable on $\Sigma$. This was observed by Dirac [8] for $k \geqslant 6$ and proved by Thomassen [36] for $k=5$. It follows that, for any surface $\Sigma$ and any integer $k \geqslant 5$, there is a polynomial-time algorithm that decides whether a graph $G$ embeddable on $\Sigma$ is $k$-colourable. For smaller values of $k$, i.e. $k \in\{2,3,4\}$, there are infinitely many $(k+1)$-critical graphs embeddable on any surface $\Sigma$ distinct from the sphere. (For $k=4$, this follows from a result of Fisk [11].) For $k=2$, it is polynomial-time solvable to decide whether a graph is 2-colourable. In contrast, deciding whether a graph embeddable on the sphere (and thus on any other surface) is 3-colourable is NP-complete. (See [15]). For $k=4$, the problem remains open, except for the sphere, for which there is a trivial algorithm by the Four Colour Theorem.

We are interested in the analogous problems for dicolouring.

\section{$\Sigma$ - $k$-DiCOLOURABILITY}

Input: A digraph $D$ embeddable on $\Sigma$.

Question: Is $D k$-dicolourable?

A natural idea is to consider $(k+1)$-dicritical digraphs. A digraph $D$ is $(\boldsymbol{k}+\mathbf{1})$ dicritical if $\vec{\chi}(D)=k+1$ and $\vec{\chi}(H) \leqslant k$ for every proper subdigraph $H$ of $D$. One easily derives from Euler's Formula that, for any $k \geqslant 7$, the number of $(k+1)$-dicritical digraphs embeddable on a surface is finite (Proposition 29). Adapting the standard method for 
critical graphs to dicritical digraphs, we prove in Corollary 33, that the number of $(k+1)$ dicritical digraphs embeddable on a surface is finite for any $k \geqslant 6$. Consequently, for any surface $\Sigma$ and any integer $k \geqslant 6$, one can solve $\Sigma$ - $k$-DiCOLOURABILITY in polynomial time.

When $k=2$, in contrast to the undirected case, Bokal et al. [5] showed that deciding whether a digraph is 2-dicolourable is NP-complete. We show (Theorem 34) that it remains NP-complete when restricted to digraphs embeddable on the sphere (and hence in any surface). In other words, $\Sigma$-2-DiCOLOURABILITY is NP-complete for any surface $\Sigma$. Since the chromatic number of $G$ is equal to the dichromatic number of the bidirected graph $\overleftrightarrow{G}$, the NP-completeness of the 3 -colourability of a graph embeddable in $\Sigma$ implies that $\Sigma$-3-Dicolourability is NP-complete for any surface $\Sigma$. The complexity of $\Sigma$ $k$-Dicolourability for $k \in\{4,5\}$ and $\Sigma$ different from the sphere remains open, see Problem 39.

We then consider the restriction of $\Sigma$ - $k$-DiCOLOURABILITY to oriented graphs.

\section{$\Sigma$-ORIENTED- $k$-DiCOLOURABILITY}

Input: An oriented $D$ embeddable on $\Sigma$.

Question: Is $D k$-dicolourable?

For any surface $\Sigma$ and any integer $k \geqslant 3$, there are only finitely many $(k+1)$-dicritical oriented graphs embeddable on $\Sigma$. For $k \geqslant 4$, this follows easily from Euler's Formula (see Proposition 9); for $k=3$, it was proved by Kostochka and Stiebitz [21] (See Theorem 10). This implies that, for any surface $\Sigma$ and any integer $k \geqslant 3$, one can solve $\Sigma$-ORIENTED$k$-Dicolourability in polynomial time. Hence we are left with the complexity of $\Sigma$ ORIENTED-2-DiCOLOURABILITY.

If Conjecture 7 is true, then $\mathbb{S}_{0}$-ORIENTED-2-DicolourabiLITY can be trivially solved in polynomial time because the answer is always positive. Conversely, we show in Theorem 35 that if Conjecture 7 is false then $\mathbb{S}_{0}$-ORIENTED-2-DicolOURABILITY is NP-complete.

\section{Preliminaries}

\subsection{Dicritical oriented graphs}

Recall that a digraph $D$ is $\boldsymbol{k}$-dicritical if $\vec{\chi}(D)=k$ and $\vec{\chi}(H)<k$ for every proper subdigraph $H$ of $D$. In this subsection, we establish some results on dicritical oriented graphs which will be useful to prove our main results.

The following proposition is well-known and easy to prove. Note that it is the only result of this section about digraphs, every other result is about oriented graphs.

Proposition 8. Let $D$ be a k-dicritical digraph. Then $d^{+}(v), d^{-}(v) \geqslant k-1$ for all $v \in V(D)$.

The next result gives an upper bound on the number of $k$-dicritical oriented graphs embeddable on a surface, when $k \geqslant 5$. This result is used to prove Theorem 21 . 
Proposition 9. Let $k \geqslant 5$ and let $\vec{G}$ be a $k$-dicritical oriented graph embedded in a surface with Euler characteristic c. Then

$$
n(\vec{G}) \leqslant \frac{-3 c}{k-4}
$$

Proof. By Proposition $8, d^{+}(v), d^{-}(v) \geqslant k-1$ for every vertex $v$ of $\vec{G}$. Moreover, by Theorem $2, \operatorname{Ad}(\vec{G}) \leqslant 6-\frac{6 c}{n(\vec{G})}$ and so $2(k-1) \leqslant 6-\frac{6 c}{n(\vec{G})}$. Hence $n(\vec{G})(k-1-3) \leqslant-3 c$ which yields the result.

Kostochka and Stieblitz [21] proved that for every 4-dicritical oriented graph $G$, $3 m(\vec{G}) \geqslant 10 n(\vec{G})-4$. It implies that the 4-dicritical oriented graphs that are embeddable on a given surface have bounded number of vertices. This result is used to prove Theorem 21 and Theorem 23.

Theorem 10 (Kostochka and Stiebitz [21]). Let $\vec{G}$ be a 4-dicritical oriented graphs embedded in a surface with Euler characteristic c. Then $n(\vec{G}) \leqslant 4-9$ c.

Proof. By Euler's formula, we have $m(\vec{G}) \leqslant 3 n(\vec{G})-3 c$ and thus $10 n(\vec{G})-4 \leqslant 3 m(\vec{G}) \leqslant$ $9 n(G)-9 c$. Therefore $n(\vec{G}) \leqslant 4-9 c$.

A graph $G$ is non-separable if it is connected and $G-v$ is connected for all $v \in V(G)$. Let $G$ be a graph. A block of $G$ is a subgraph which is non-separable and is maximal with respect to this property. A cactus is a graph whose blocks are cycles or edges. A directed cactus is an oriented graph whose blocks are directed cycles or arcs. In other words, a directed cactus is an oriented cactus in which every cycle is directed. The following result is the main tool in the proofs of Theorem 23 and Theorem 27. We state it here for oriented graphs, but a more general version holding for digraphs exists and is used latter in the paper, see Theorem 30 .

Theorem 11 (Bang-Jensen et al. [3]). Let $\vec{G}$ be a $k$-dicritical oriented graph. The subdigraph induced by the vertices of in- and out-degree $k-1$ is a directed cactus.

The next two results are technical lemmas on cacti that we use to prove Theorem 23 and Theorem 27.

Lemma 12. Let $G$ be a cactus. Then $m(G) \leqslant \frac{3}{2}(n(G)-1)$. Moreover, equality holds if and only if $G$ is connected and every block is a triangle.

Proof. We proceed by induction on $n(G)$ the number of vertices of $G$, the result being trivial if $n(G) \leqslant 2$.

Suppose now that $n(G)>2$. If $G$ is not connected, then applying the induction hypothesis on each connected component, and summing the obtained inequalities give the result.

Suppose now that $G$ is connected. If it is a cycle or an edge, then $m(G) \leqslant n(G)$ so the result holds. If $G$ is not a cycle, then $G$ contains a leaf block $C$ with attachment $x$. 
Let $G^{\prime}$ be the graph obtained from $G$ by deleting all vertices of $C$ except $x$. We have $n\left(G^{\prime}\right)=n(G)-n(C)+1$ and $m\left(G^{\prime}\right)=m(G)-m(C)$ and $m(C)=n(C)-\epsilon(C)$, with $\epsilon(C)=1$ if and only if $C$ is an edge, and $\epsilon(C)=0$ otherwise. By the induction hypothesis, $m\left(G^{\prime}\right) \leqslant \frac{3}{2}\left(n\left(G^{\prime}\right)-1\right)$. Hence

$$
\begin{aligned}
m(G) & \leqslant \frac{3}{2}(n(G)-n(C)+1-1)+n(C)-\epsilon(C) \\
& =\frac{3}{2}(n(G)-1)-\frac{1}{2} n(C)+\frac{3}{2}-\epsilon(C) \\
& \leqslant \frac{3}{2}(n(G)-1)
\end{aligned}
$$

Moreover, $-\frac{1}{2} n(C)+\frac{3}{2}-\epsilon(C)=0$ if and only if $C$ is a triangle, which implies that the bound is tight if and only if all blocks are triangles.

Lemma 13. Every cactus of order $n$ contains an induced forest of order $\left\lceil\frac{2}{3} n\right\rceil$.

Proof. By induction on $n$. Let $G$ be a cactus of order $n$. If $G$ is not connected, then we have the result by applying the induction on each connected component and summing the obtained inequalities.

If $G$ is a cycle or an edge, the result is clear. If not, then $G$ admits a leaf block $C$ with attachment $x$. Let $G^{\prime}$ be the graph obtained from $G$ by removing all vertices of $V(C)$ except $x$. By the induction hypothesis, $G^{\prime}$ contains a set $X$ of order at least $\left\lceil\frac{2}{3}(n-n(C)+1)\right\rceil$ such that the induced subgraph $G\langle X\rangle$ is a forest. Let $y$ be a vertex of $V(C) \backslash\{x\}$. If $C$ is an edge, then $G\langle X \cup\{y\}\rangle$ is a forest of order at least $\left\lceil\frac{2}{3}(n-n(C)+\right.$ $1)\rceil+1 \geqslant\left\lceil\frac{2}{3} n\right\rceil$. If $C$ is a cycle, then $G\langle X \cup V(C) \backslash\{y\}\rangle$ is a forest of order at least $\left\lceil\frac{2}{3}(n-n(C)+1)\right\rceil+n(C)-1 \geqslant\left\lceil\frac{2}{3} n\right\rceil$ because $n(C) \geqslant 3$.

\subsection{Large acyclic subdigraphs and dichromatic number in oriented graphs}

Note that, in a tournament, the induced acyclic subdigraphs are the transitive subtournaments. The following classic result of Stearns is used to prove Proposition 20.

Lemma 14 (Stearns [34]). Every tournament of order $n$ has an induced acyclic subdigraph of order $\left\lfloor\log _{2} n\right\rfloor+1$.

Erdős and Moser [10] proved that, for every $n \geqslant 2$, there exists a tournament of order $n$ whose largest acyclic subdigraph has at most $\left\lfloor 2 \log _{2} n\right\rfloor+1$ vertices. Since the dichromatic number of an $n$-vertex digraph is at least $n$ divided by the order of a largest acyclic induced subdigraph, we get the following, used to prove Theorem 21.

Proposition 15 (Erdős and Moser [10]). For every $n \geqslant 2$, there exists a tournament $T$ of order $n$ such that $\vec{\chi}(T) \geqslant \frac{n}{2 \log (n)+1}$.

We now turn our attention to acyclic subdigraphs in small digraphs. A digraph is $\boldsymbol{k}$-diregular if all its vertices have in- and out-degree $k$. The next two results are used to prove Theorem 27. 
Theorem 16 (Reid and Parker [30]; Sanchez-Flores [33]).

(i) Every tournament of order 14 has a transitive subtournament of order 5 .

(ii) There is a unique tournament $S T_{13}$ of order 13 with no transitive subtournament of order 5. This tournament $S T_{13}$ is 6-diregular.

(iii) There is a unique tournament $S T_{12}$ of order 12 with no transitive subtournament of order 5 . This tournament is obtained from $S T_{13}$ by removing any vertex.

\section{Corollary 17.}

(i) The only oriented graph of order 13 with no acyclic induced subdigraph of order 5 is $S T_{13}$.

(ii) Every oriented graph of order 12 with no acyclic induced subdigraph of order 5 satisfies $d^{+}(v), d^{-}(v) \geqslant 5$ for every vertex $v$. In particular it has at least 60 arcs.

Proof. (i) Let $\vec{G}$ be an oriented graph of order 13 distinct from $S T_{13}$. If $\vec{G}$ is a tournament, then we have the result by Theorem 16 (ii). Assume now that $\vec{G}$ is not a tournament.Then there exist non-adjacent vertices $x, y$ in $\vec{G}$. We add arcs to $\vec{G}$ to obtain a tournament $T$ in which $x$ does not have in-degree 6 . By Theorem 16 (ii), $T$ has a transitive subtournament of order 5 , and thus $\vec{G}$ has an acyclic induced subdigraph of order 5 .

(ii) Let $\vec{G}$ be an oriented graph of order 12 . Assume that there is a vertex $v$ with out-degree at most 4 in $\vec{G}$. We add arcs to $\vec{G}$ to obtain a tournament $T$ in which $x$ has the same out-degree as in $\vec{G}$. Now $T \neq S T_{12}$ because every of $S T_{12}$ has out-degree 5 and 6. Hence, by Theorem 16 (ii), $T$ has a transitive subtournament of order 5 , and thus $\vec{G}$ has an acyclic induced subdigraph of order 5 .

Similarly, if there is a vertex $v$ with out-degree at most 4 , then $\vec{G}$ has an acyclic induced subdigraph of order 5 .

\subsection{Small oriented graphs of dichromatic number 3 or 4}

We describe the 3- and 4-dichromatic oriented graphs with a given number of vertices. These results are used in the proof of Theorem 22, Theorem 23 and Theorem 27.

Proposition 18. (i) All oriented graphs on at most 6 vertices are 2-dicolourable.

(ii) The unique smallest 3-dicritical oriented graph on 7 vertices has 20 arcs.

(iii) The unique smallest 3-dicritical oriented graph on 8 vertices has 21 arcs.

(iv) The unique smallest 3-dicritical oriented graph on 9 vertices has 23 arcs.

(v) Every 3-dicritical oriented graph on at least 10 vertices has at least 21 arcs. 
Proof. (i) was shown in [29].

We verified (ii), (iii), and (iv) by exhaustive computation. By Proposition 8, 3dicritical oriented graphs have minimum in- and out-degree at least 2 and thus their underlying graph have minimum degree 4 . We used the program nauty [27] to generate all (non-oriented) graphs of order 7,8 and 9 of minimum degree at least 4 . From this list, we extracted the graphs of arboricity at least 3 and generated the orientations of minimum in- and out-degree at least 2 using McKay's program nauty [27]. Finally, we kept only the orientations that were 3-dicritical. The code can be found at https://www . combinatorics.org/ojs/index.php/eljc/article/view/v29i1p30/code or https:// github.com/ClementRambaud/cdicoloring.

To prove $(v)$, observe that 3-dicritical oriented graphs have in- and out-degree at least 2 (Proposition 8) and that Theorem 11 implies that a 3-dicritical oriented graph cannot be 2-diregular.

Theorem 19 (Neumann Lara [29]). All oriented graphs on at most 10 vertices are 3dicolourable. The unique smallest oriented graph with dichromatic number 4 on 11 vertices has 55 edges. It is depicted in Figure 1.

Proof. In [29], Neumann-Lara proved that every tournament (and so oriented graph) of order 10 is 3-dicolourable, and that there is a unique tournament $S T_{11}$ with order 11 and dichromatic number 4. It is depicted in Figure 1. Let us show that this tournament is dicritical, which implies the result.

Since $S T_{11}$ is arc-transitive (for any two arcs $e$ and $e^{\prime}$ there is an automorphism sending $e$ onto $e^{\prime}$ ), it suffices to show an arc $e$ such that $T \backslash e$ is 3-dicolourable. Let us use the vertex numbering of Figure 1. Observe that in $T \backslash(4,2)$, the set $S=\{0,1,2,4,5\}$ induces an acyclic subdigraph of order 5 . Now $S T_{11}-S$ has 6 vertices and is 2-dicolourable by (i). Hence $T \backslash(4,2)$ is 3 -dicolourable and $T$ is 4 -dicritical.

In [29] it is stated without proof that all oriented graphs on at most 16 vertices are 4-dicolourable. Here, we prove a weaker statement that suffices for our purposes.

Proposition 20. All oriented graphs on at most 15 vertices are 4-dicolourable.

Proof. Let $\vec{G}$ be an oriented graph of order $n \leqslant 15$.

Assume first $n=15$. According to Theorem $16(i), \vec{G}$ contains an acyclic subdigraph $S$ of order 5 . Now $\vec{G}-S$ has 10 vertices, and so is 3 -dicolourable by Theorem 19 . Thus $\vec{G}$ is 4-dicolourable.

Assume now that $13 \leqslant n \leqslant 14$. Then $\vec{G}$ contains an acyclic subdigraph $S$ of order $\left\lfloor\log _{2}(13)\right\rfloor+1=4$ by Lemma 14 . Now $\vec{G}-S$ has 10 vertices, and so is 3 -dicolourable by Theorem 19. Thus $\vec{G}$ is 4-dicolourable.

Assume now that $n \leqslant 12$. Let $(A, B)$ be a partition of $\vec{G}$ with $|A|=|B|=6$. By Proposition 18, each of $\vec{G}\langle A\rangle$ and $\vec{G}\langle A\rangle$ is 2-dicolourable. Hence, $\vec{G}$ is 4-dicolourable. 


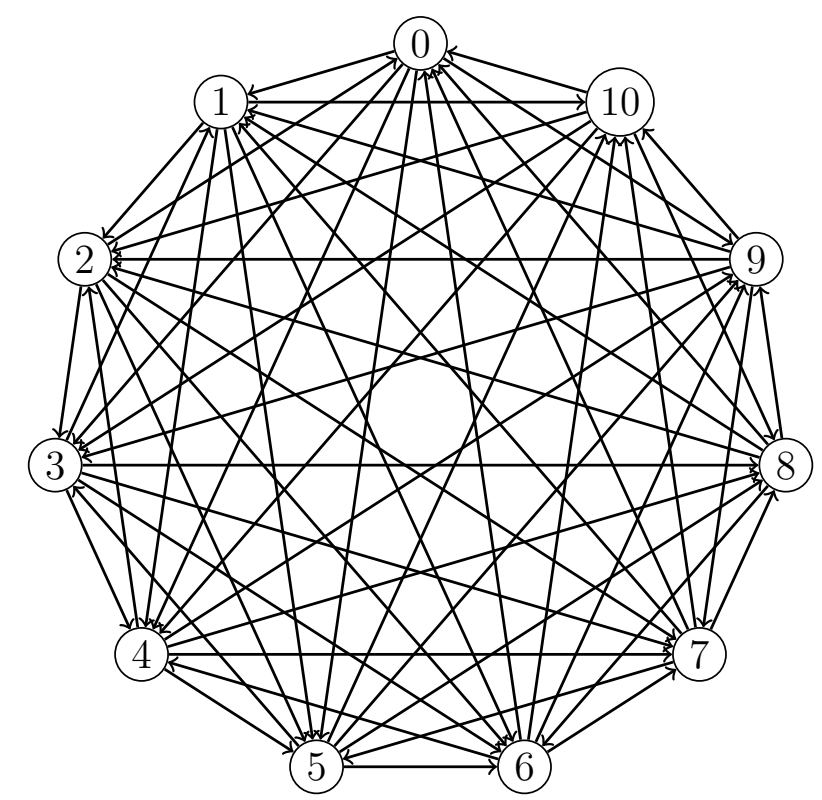

Figure 1: The tournament $S T_{11}$.

\section{Dichromatic number of surfaces}

\subsection{General bounds}

We first determine the asymptotic behaviour of the dichromatic number of a surface of given Euler characteristic. The upper bound was pointed out to us by Raphael Steiner.

Theorem 21. There exist two constants $a_{1}$ and $a_{2}$ such that, for every surface $\Sigma$ with Euler characteristic $c \leqslant-2$, we have

$$
a_{1} \frac{\sqrt{-c}}{\log (-c)} \leqslant \vec{\chi}(\Sigma) \leqslant a_{2} \frac{\sqrt{-c}}{\log (-c)}
$$

Proof. Let us first establish the lower bound. By Proposition 15, there exists a tournament $T$ of order $H(c)$ such that $\vec{\chi}(T) \geqslant \frac{H(c)}{2 \log H(c)+1}$. But, by Theorem 4, this tournament is embeddable on $\Sigma$. So $\vec{\chi}(\Sigma) \geqslant \vec{\chi}(T) \geqslant \frac{H(c)}{2 \log H(c)+1}$. Since $H(c)=\left\lfloor\frac{7+\sqrt{49-24 c}}{2}\right\rfloor$, we get the lower bound.

To see the upper bound, let $\Sigma$ be a surface of Euler characteristic $c$ and dichromatic number $k \geqslant 4$. (We will choose the constant $a_{2}$ large enough to not care about smaller values of $k$.) Let $\vec{G}$ be a $k$-dicritical oriented graph with $n$ vertices and $m$ arcs embedded in $\Sigma$.

By Proposition 9 and Theorem 10 there is a constant $b_{1}$ such that $n \leqslant-b_{1} c$. Thus, Euler's Formula implies that there is a constant $b_{2}$ such that $m \leqslant-b_{2} c$. Now, by a result of [9], there is a constant $b_{3}$ such that $m \geqslant b_{3} k^{2} \log ^{2}(k)$. This yields $b_{4}$ such that, $k \leqslant b_{4} \frac{\sqrt{-c}}{\log (k)}$. Applying the lower bound $a_{1} \frac{\sqrt{-c}}{\log (-c)} \leqslant k$, we get that there is a $b_{5}$ such that $\log (k) \geqslant b_{5} \log (-c)$. This yields the upper bound. 


\subsection{Projective plane, torus, Klein bottle, and Dyck's surface}

We begin with a lower bound.

Theorem 22. If $\Sigma$ is a surface of Euler characteristic at most 1 , then $\vec{\chi}(\Sigma) \geqslant 3$.

Proof. We show $\vec{\chi}\left(\mathbb{N}_{1}\right), \vec{\chi}\left(\mathbb{N}_{2}\right), \vec{\chi}\left(\mathbb{S}_{1}\right) \geqslant 3$. To prove the lower bound for $\mathbb{N}_{1}$, we construct an oriented graph embeddable on $\mathbb{N}_{1}$ with dichromatic number 3 .

The complete graph on 6 vertices $K_{6}$ can be embedded as a triangulation of the projective plane, that is is an embedding of $K_{6}$ in the projective plane such that all faces are triangles. Let $T$ be the orientation of $K_{6}$ displayed on the left of Figure 2. Let $\vec{G}$
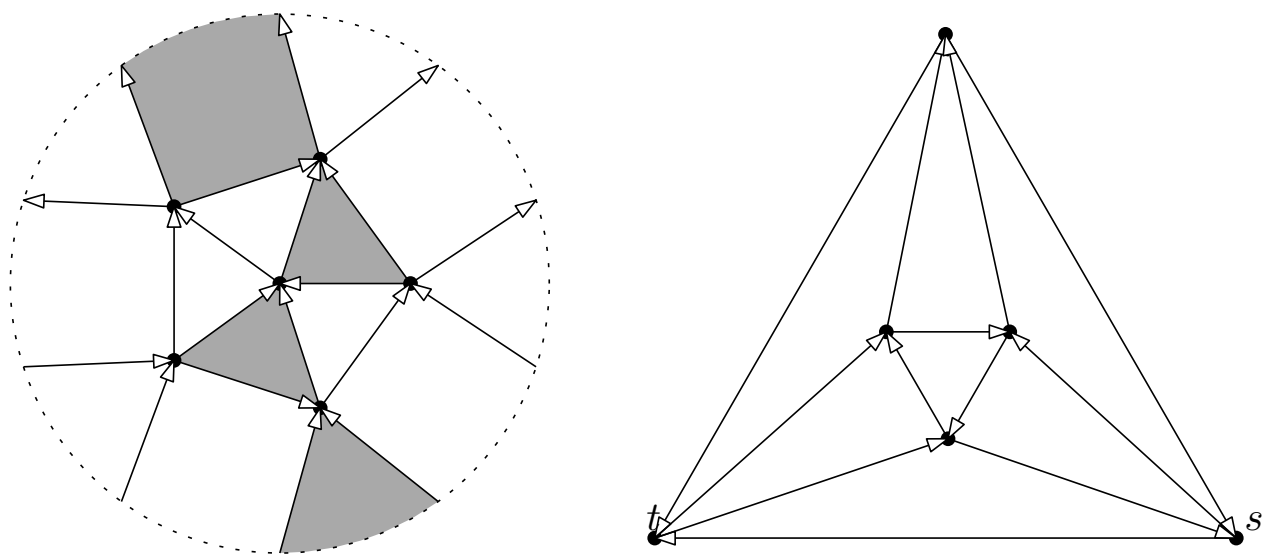

Figure 2: Left: an orientation $T$ of $K_{6}$ on the projective plane. Right: the gadget graph.

be the oriented graph obtained from $T$ by adding in each gray triangular face (which is a transitive tournament on three vertices with source $s$ and sink $t$ ), the gadget graph depicted on the left of Figure 2. Observe that in any 2-dicolouring of the gadget graph, the vertices of the outer face do not have all the same colour.

Assume now for a contradiction that $\vec{G}$ admits a 2-dicolouring. Observe that either we have a monochromatic directed triangle in $T$ or one of the gray triangles is monochromatic. But then the 2-dicolouring cannot be extended to the gadget inside this transitive tournament by the above observation. Hence $\vec{G}$ is not 2 -dicolourable.

While the above graph has 15 vertices, for the Klein bottle and the torus we also have orientations of $K_{7} \backslash e$ and $K_{7}$, respectively, of dichromatic number 3, see Proposition 18 (ii).

Since any surface different from the sphere admits an embedding of one of the above graphs, we get the result.

Let us continue with an upper bound.

Theorem 23. $\vec{\chi}\left(\mathbb{N}_{3}\right) \leqslant 3$.

Proof. Suppose for a contradiction that there exists a 4-dicritical oriented graph $\vec{G}$ embeddable on $\mathbb{N}_{3}$. By Theorem 19, $12 \leqslant n(\vec{G})$ (because $S T_{11}$ is an orientation of $K_{11}$ which is not embeddable on $\mathbb{N}_{3}$ ), and by Theorem $10, n(\vec{G}) \leqslant 13$ (because $c\left(\mathbb{N}_{3}\right)=-1$ ), 
Let $T$ be the subdigraph induced by the vertices of degree 6 (i.e. in-degree 3 and outdegree 3$)$. Let $H=G-T$, and let $m(H, T)$ be the number of arcs with one end-vertex in $H$ and the other in $T$. By Theorem 11, $T$ is a directed cactus. In particular, it has a vertex with at most two neighbours in $T$ and thus at least 4 in $H$. Hence $n(H) \geqslant 4$.

Set $\varepsilon(H)=\sum_{v \in V(H)}(d(v)-7)$. Euler's Formula yields $6 n(T)+7(n(\vec{G})-n(T))+\varepsilon(H)=$ $2 m(\vec{G}) \leqslant 6 n+6$ and so:

$$
n(T) \geqslant n(\vec{G})-6+\varepsilon(H) \geqslant n(\vec{G})-6 .
$$

By Lemma $12, m(T) \leqslant \frac{3}{2} n(T)-\frac{3}{2}$. Hence

$$
m(H, T)=6 n(T)-2 m(T) \geqslant 3 n(T)+3 .
$$

Since $T$ is a cactus, it is 2-dicolourable, so $H$ must have dichromatic number at least 2 , and thus $m(H) \geqslant 3$. Now

$$
\begin{aligned}
\varepsilon(H) & =m(H, T)+2 m(H)-7 n(H) \\
& \geqslant 3 n(T)+9-7 n(H)
\end{aligned}
$$

Assume for a contradiction that $n(H)=4$. Then $n(T) \geqslant 8$. By (4), we have $\varepsilon(H) \geqslant 5$. Thus by $(2), n(T) \geqslant n(\vec{G})-1$, a contradiction. Hence $n(H) \in\{5,6\}$.

All together, we have:

$$
n(\vec{G}) \in\{12,13\}, \quad n(T) \in\{6,7,8\} \quad \text { and } \quad n(H) \in\{5,6\}
$$

We are now going to bound the number of edges in $H$ :

$$
\begin{aligned}
& m(H)=m(G)-m(H, T)-m(T) \\
& =m(G)-(6 n(T)-2 m(T))-m(T) \\
& =m(G)-6 n(T)+m(T) \\
& \leqslant 3 n(\vec{G})+3-6 n(T)+\frac{3}{2}(n(T)-1) \quad \text { by Euler's formula and Lemma } 12 \\
& \leqslant 3 n(\vec{G})+3-\frac{9}{2} n(T)-\frac{3}{2} \\
& \leqslant 3 n(\vec{G})+3-\frac{9}{2}(n(\vec{G})-6)-\frac{3}{2} \\
& \leqslant 10
\end{aligned}
$$

Let $A$ be a maximum acyclic subdigraph of $T$. By Lemma $13, n(A) \geqslant\left\lceil\frac{2}{3} n(T)\right\rceil$. So $n(T-A) \leqslant \frac{1}{3} n(T) \leqslant 2$ by $(5)$, and since $n(H) \leqslant 6$, we have $n(\vec{G}-A) \leqslant 6+2=8$. Each vertex $v$ in $T-A$ must be in a cycle in $T\langle V(A) \cup\{v\}\rangle$, and so has at most 4 neighbours in $\vec{G}-A$. Hence $m(\vec{G}-A) \leqslant m(H)+8 \leqslant 18$. Now, $\vec{G}-A$ is 2 -dicolourable by Proposition 18 and thus $\vec{G}$ is 3-dicolourable.

Combining Theorems 22 and 23 with the fact that Dyck's surface is the torus plus a cross-cap determines the dichromatic number of the above surfaces.

Corollary 24. $\vec{\chi}\left(\mathbb{N}_{1}\right)=\vec{\chi}\left(\mathbb{N}_{2}\right)=\vec{\chi}\left(\mathbb{N}_{3}\right)=\vec{\chi}\left(\mathbb{S}_{1}\right)=3$. 


\subsection{The dichromatic number of $\mathbb{S}_{5}$ and $\mathbb{N}_{10}$}

By Theorem 4, the complete graph on 11 vertices is embeddable on every surface of Euler characteristic at most -8 and by Theorem 19 its orientation $S T_{11}$ has dichromatic number 4. Hence we have the following.

Proposition 25. If $\Sigma$ is a surface of Euler characteristic at most -8 , then $\vec{\chi}(\Sigma) \geqslant 4$.

The remainder of the subsection is dedicated to the proof that the above inequality is tight for $\mathbb{S}_{5}$ and $\mathbb{N}_{10}$.

We shall need some preliminary notions and results. Let $D$ be a digraph. A list assignment of $D$ is a mapping $L: V(D) \rightarrow \mathcal{P}(C)$, where $C$ is a set of colours. An $\boldsymbol{L}$-dicolouring of $D$ is a dicolouring $\phi$ of $G$ such that $\phi(v) \in L(v)$ for all $v \in V(D)$. If $D$ admits an $L$-dicolouring, then it is $\boldsymbol{L}$-dicolourable.

Theorem 26 (Harutyunyan and Mohar [18]). Let $D$ be a digraph and $L$ be a list assignment of $D$ such that $|L(v)| \geqslant \max \left\{d^{+}(v), d^{-}(v)\right\}$ for every vertex $v \in V(D)$. If $D$ is not $L$-dicolourable, then $|L(v)|=\max \left\{d^{+}(v), d^{-}(v)\right\}$ for every vertex $v$ and every block of $D$ is either

- a directed cycle, or

- a bidirected odd cycle, or

- a bidirected complete graph.

The following result shows that in fact equality holds.

Theorem 27. Every oriented graph embeddable on $\mathbb{S}_{5}$ or $\mathbb{N}_{10}$ is 4-dicolourable.

Proof. Let $\vec{G}$ be a 5 -dicritical oriented graph of order $n$ which is embedded in $\mathbb{S}_{5}$ or $\mathbb{N}_{10}$, and assume for a contradiction that $\vec{G}$ is not 4-dicolourable.

Let $T$ be the subdigraph induced by the vertices of degree 8 (i.e. in-degree 4 and out-degree 4). Set $H=\vec{G}-T, n_{8}=n(T)$ and let $m(H, T)$ be the number of arcs with one end-vertex in $H$ and the other in $T$. By Theorem 11, $T$ is a directed cactus and so is 2-dicolourable. Therefore $H$ is not 2-dicolourable. In particular, by Proposition 18, $m(H) \geqslant 20$. so:

Euler's Formula yields $8 n_{8}+9\left(n-n_{8}\right)+\sum_{v \in V(H)}(d(v)-9)=2 m(\vec{G}) \leqslant 6 n+48$ and

$$
n_{8} \geqslant 3(n-16)+\sum_{v \in V(H)}(d(v)-9) \geqslant 3(n-16)
$$

On the other hand, we have $\sum_{v \in V(T)} d(v)=8 n_{8}=2 m(T)+m(H, T)$ and $m(\vec{G})=$ $m(H)+m(H, T)+m(T)$. We deduce

$$
m(H)=m(\vec{G})+m(T)-8 n_{8}
$$


By Lemma $12, m(T) \leqslant \frac{3}{2}\left(n_{8}-1\right)$. Thus $20 \leqslant m(H) \leqslant m(\vec{G})+\frac{3}{2}\left(n_{8}-1\right)-8 n_{8}$. Hence $13 n_{8} \leqslant 2 m(\vec{G})-43$. With Eq. (6) and Euler's formula, it implies

$$
3(n-16) \leqslant n_{8} \leqslant \frac{2 m(\vec{G})-43}{13} \leqslant \frac{6 n+5}{13}
$$

After simplifying, we get $n \leqslant 19$. Moreover, by Proposition 20 , we have $n \geqslant 16$. We now distinguish few cases depending on the number $n$ of vertices.

Case $n=19$ : By Eq. (8), we have $9 \leqslant n_{8} \leqslant \frac{119}{13}$ and so $n_{8}=9$.

Assume first that $m(T)=\frac{3}{2}\left(n_{8}-1\right)=12$. By Lemma 12, $T$ is connected and each block of $T$ is a directed triangle. So $T$ is Eulerian, i.e. $d_{T}^{+}(v)=d_{T}^{-}(v)$ for all $v \in V(T)$.

Since $n_{8}=9$, then $n(H)=10$. So, by Theorem $19, H$ admits a 3-dicolouring $\phi$ with colour set $\{1,2,3\}$. Since all blocks of $T$ are directed triangles, $T$ contains a vertex $v$ such that $d_{T}^{+}(v)=d_{T}^{-}(v)=1$. So $v$ has 3 out-neighbours in $H$. Let $v_{1}, v_{2}$ be two of these out-neighbours. Let us recolour $v_{1}$ and $v_{2}$ by setting $\phi\left(v_{1}\right)=\phi\left(v_{2}\right)=4$ (since there is no digon, the resulting colouring is still proper). We then define for every vertex $x$ of $T$ :

$$
L(x)=\{1,2,3,4\} \backslash \phi\left(N^{+}(x) \cap V(H)\right)
$$

Observe that an $L$-colouring of $T$ extends the 4-colouring of $H$ into a 4-colouring of $G$, so $T$ is not $L$-colourable. Observe that $|L(x)| \geqslant 4-\left(4-d_{T}^{+}(x)\right)=\max \left\{d_{T}^{+}(x), d_{T}^{-}(x)\right\}$ because $T$ is Eulerian. Moreover, since $v_{1}$ and $v_{2}$ are both coloured $4,|L(v)| \geqslant 2=$ $\max \left\{d_{T}^{+}(x), d_{T}^{-}(x)\right\}+1$. So $T$ is $L$-dicolourable by Theorem 26 , a contradiction.

Therefore we have $m(T) \leqslant 11$. By Euler's Formula, $m(\vec{G}) \leqslant 3 n+24$, and by Eq. (7) $m(H)=m(\vec{G})-8 n_{8}+m(T)$. Hence $m(H) \leqslant 20$. But $H$ is not 2-dicolourable, so it contains a 3 -dicritical oriented subgraph $\tilde{H}$, and $m(\tilde{H}) \leqslant 20$. By Proposition 18, there is a unique such 3-dicritical oriented graph and it has 7 vertices and 20 arcs. Hence $n(\tilde{H})=7, m(\tilde{H})=m(H)=20$ and $H$ is the disjoint union of $\tilde{H}$ and a stable set $S^{\prime}$ of size 3. Observe that each vertex of $S^{\prime}$ has degree at least 9, which implies that they are adjacent to every vertex of $T$ and have degree exactly 9 .

Now, $m(\tilde{H})<m\left(K_{7}\right)$, so there are two non-adjacent vertices $x, y$ in $\tilde{H}$. Thus $S=$ $S^{\prime} \cup\{x, y\}$ is a stable set of order 5 in $H$. Moreover, by Lemma 13, $T$ has an acyclic subdigraph $A$ of order 6 . Pick $v \in V(T) \backslash V(A)$. The subdigraph $B$ of $\vec{G}$ induced by $S \cup\{v\}$ is acyclic and has order 6 . Let $G^{\prime}=\vec{G}-(A \cup B)$. Observe that $G^{\prime}$ has order $19-6-6=7$. Recall that by Theorem 8 , oriented graphs on at most 6 vertices are 2-dicolourable.

Let $w \in V\left(G^{\prime}\right) \cap V(T)$.

- If $|N(w) \cap V(A)| \leqslant 1$, then the subdigraph $A^{\prime}$ induced by $V(A) \cup\{w\}$ is acyclic. Hence $G$ can be partitioned into two acyclic subdigraphs $A^{\prime}$ and $B$ and $G-A^{\prime} \cup B$ which has order 6 and so is 2-dicolourable. Thus $\vec{G}$ is 4 -dicolourable, a contradiction.

- If $|N(w) \cap V(A)| \geqslant 2$, then as $w$ is adjacent to all vertices of $S^{\prime}$, we have $d_{G^{\prime}}(w) \leqslant$ $8-2-3=3$. Now, $G^{\prime}-\{w\}$ is 2-dicolourable, and since $d_{G^{\prime}}(w)=3, G^{\prime}$ is also 2-dicolourable, and thus $G$ is 4-dicolourable, a contradiction. 
Case $n=18$ : By Eq. (8), we have $n_{8} \geqslant 6$. Let $u$ be a vertex of degree 8 in $\vec{G}$ and consider $\vec{G}^{\prime}=\vec{G}-\left(N^{+}(u) \cup\{u\}\right)$ which is of order 13. By Theorem $2, \operatorname{Ad}\left(\vec{G}^{\prime}\right) \leqslant 6+\frac{6 \times 8}{13}<12=$ $\operatorname{Ad}\left(S T_{13}\right)$. So $\vec{G}^{\prime} \neq S T_{13}$, and so, by Theorem $16, \vec{G}^{\prime}$ has an acyclic subdigraph $A_{0}$ of order 5 . Then the subdigraph $A$ of $\vec{G}$ induced by $V\left(A_{0}\right) \cup\{u\}$ is acyclic and has order 6 .

Set $B=\vec{G}-A$. Then $m(B)=m(\vec{G})-\sum_{v \in V(A)} d(v)+m(A) \leqslant 78-8 \times 6+m(A) \leqslant$ $30+m(A) \leqslant 30+\left(\begin{array}{c}6 \\ 2\end{array}\right)=45$. Moreover, $B$ is not 3 -dicolourable, for otherwise $\vec{G}$ would be 4-dicolourable. Hence $B$ contains a 4-dicritical subdigraph $\tilde{B}$. Because $m(\tilde{B}) \leqslant$ $m(B) \leqslant 45<55=m\left(S T_{11}\right)$, the oriented graph $\tilde{B}$ is not $S T_{11}$. Thus $\tilde{B}$ has order 12 by Theorem 19. Consequently, for every vertex $v$ of $B, B-v$ is 3-dicolourable and the subdigraph induced by $V(A) \cup\{v\}$ is not acyclic for otherwise $\vec{G}$ would be 4 -dicolourable. Hence, for each $v \in B, v$ must have at least one in-neighbour and one out-neighbour in $A$ and therefore $m(A, B) \geqslant 2 n(B)=24$.

But then $m(B)=m(\vec{G})-m(A, B)-m(A) \leqslant 54-m(A)$. Recall that $m(B) \leqslant$ $30+m(A)$. Thus

$$
m(B) \leqslant \frac{1}{2}((30+m(A))+(54-m(A)))=42 .
$$

We now do a similar reasoning with $B$ as the one we just did with $\vec{G}$. Because $m(B) \leqslant 42<60$, by Corollary 17 (ii), $B$ has an acyclic subdigraph $A^{\prime}$ of order 5 . Set $B^{\prime}=B-A^{\prime}$. Then $B^{\prime}$ is not 2-dicolourable for otherwise $B$ would be 3-dicolourable. Recall that $|\tilde{B}|=|B|$ so $d_{B}^{+}(v), d_{B}^{-}(v) \geqslant 3$ for all $v \in V(B)$ by Proposition 8 . Thus $m\left(B^{\prime}\right)=m(B)-\sum_{v \in V\left(A^{\prime}\right)} d_{B}(v)+m\left(A^{\prime}\right) \leqslant 42-6 \times 5+m\left(A^{\prime}\right)=12+m\left(A^{\prime}\right)$.

Moreover $B^{\prime}$ has order 7 . Thus, by Theorem $19, B^{\prime}-v$ is 2-dicolourable for all vertex $v$ of $B^{\prime}$. Therefore $\left|N(v) \cap V\left(A^{\prime}\right)\right| \geqslant 2$ for otherwise $B$ would be 3 -dicolourable. Hence $m\left(A^{\prime}, B^{\prime}\right) \geqslant 2 n\left(B^{\prime}\right)=14$. Consequently $m\left(B^{\prime}\right)=m(B)-m\left(A^{\prime}, B^{\prime}\right)-m(A) \leqslant 28-m\left(A^{\prime}\right)$. Together with $m\left(B^{\prime}\right) \leqslant 12+m\left(A^{\prime}\right)$, this yields

$$
m\left(B^{\prime}\right) \leqslant \frac{1}{2}\left(\left(12+m\left(A^{\prime}\right)\right)+\left(28-m\left(A^{\prime}\right)\right)\right)=20 .
$$

By Proposition $18, B^{\prime}$ is uniquely determined and has exactly 20 arcs. Thus there are five vertices with degree 6 in $B^{\prime}$, and two with degree 5 . Moreover, each vertex of $B^{\prime}$ has at least two neighbours in $A^{\prime}$ and two neighbours in $A$. Hence, five vertices of $B^{\prime}$ have degree at least 10 in $G$, and two have degree at least 9 in $G$. Let us denote by $n_{9}$ and $n_{\geqslant 10}$ the number of vertices of degree 9 and at least 10 , respectively. We have

$$
2 m(G)=2 \times 78=8 \times 11+9 \times 2+10 \times 5 \leqslant 8 n_{8}+9 n_{9}+10 n_{\geqslant 10} \leqslant 2 m(\vec{G})=2 \times 78
$$

We deduce that the degree list of the vertices of $\vec{G}$ : there are eleven vertices with degree 8, two vertices with degree 9 and five with degree 10. But this contradicts Eq. (8) which states that $\vec{G}$ has at most eight vertices of degree 8 .

Case $n=17$ : We have $n_{8} \geqslant 3(17-16)=3$. Let $u$ be a vertex of degree 8 . Recall that

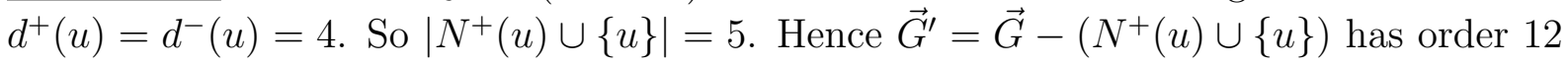


and $m\left(\vec{G}^{\prime}\right)=m(\vec{G})-\sum_{v \in N^{+}(u) \cup\{u\}} d(v)+m\left(N^{+}(u) \cup\{u\}\right)$. But $m(\vec{G}) \leqslant 3 n+24=75$, $\sum_{v \in N^{+}(u) \cup\{u\}} d(v) \geqslant 5 \times 8$, and $m\left(N^{+}(u) \cup\{u\}\right) \leqslant\left(\begin{array}{l}5 \\ 2\end{array}\right)=10$. Therefore $m\left(\vec{G}^{\prime}\right) \leqslant 75-40+$ $10=45<60$. Thus, by Corollary $17, \vec{G}^{\prime}$ has an acyclic subdigraph of order 5 . Adding $u$ to this subdigraph, we obtain an acyclic subdigraph $A$ of order 6 . Set $B=\vec{G}-A$. Then $n(B)=11$ and $m(B)=m(\vec{G})-\sum_{v \in V(A)} d(v)+m(A) \leqslant 75-6 \times 8+\left(\begin{array}{l}6 \\ 2\end{array}\right)=42$. Hence $B \neq S T_{11}$ and so $B$ is 3 -dicolourable by Theorem 19 (ii). This implies that $\vec{G}$ is 4-dicolourable, a contradiction.

Case $n=16$ : By Theorem 16, $\vec{G}$ has an acyclic subdigraph $A$ of order 5 . Set $B=\vec{G}-A$. We have $n(B)=11$.

If $B$ is not $S T_{11}$, then by Theorem 19 (ii), it is 3-dicolourable, and thus $\vec{G}$ is 4 dicolourable, a contradiction.

Henceforth $B=S T_{11}$, so $m(B)=55$. We have $m(\vec{G}) \leqslant 3 n+24=72$. Thus $m(A, B) \leqslant m(A, B)+m(A)=m(\vec{G})-m(B) \leqslant 72-55=17$. But $\frac{17}{11}<2$, so there is a vertex $v$ of $B$ such that $|N(v) \cap V(A)| \leqslant 1$. The subdigraph $A^{\prime}$ induced $V(A) \cup\{v\}$ is then acyclic and of order 6 . The oriented graph $\vec{G}-A^{\prime}$ has order 10 , so, by Theorem 19 (ii), it is 3-dicolourable. Thus $\vec{G}$ is 4-dicolourable. This contradiction completes the proof.

Clearly, Theorem 27 also provides an upper bound for the dichromatic number of surfaces of higher Euler characteristic. Moreover, Proposition 25 and Theorem 27 allow to determine the following dichromatic numbers precisely.

Corollary 28. $\vec{\chi}\left(\mathbb{N}_{10}\right)=\vec{\chi}\left(\mathbb{S}_{5}\right)=4$.

\subsection{Dicritical digraphs embeddable in a fixed surface}

The goal of this section is to prove that for any surface $\Sigma$, there is a finite number of $(k+1)$-dicritical digraphs embeddable on $\Sigma$ for every $k \geqslant 6$, see Corollary 33 .

The following result bounds the number of $k$-dicritical digraphs embeddable on a surface, when $k \geqslant 8$.

Proposition 29. Let $k \geqslant 8$ and let $D$ be a $k$-dicritical digraph embedded in a surface with Euler characteristic c. Then

$$
n(D) \leqslant \frac{-6 c}{k-7}
$$

Proof. By Proposition $8, d^{+}(v), d^{-}(v) \geqslant k-1$ for every vertex $v$ of $D$. Moreover, since there are at most two arcs between any two vertices, by Theorem $2, \operatorname{Ad}(D) \leqslant 12-\frac{12 c}{n(D)}$ and so $2(k-1) \leqslant 12-\frac{12 c}{n(D)}$. Now $n(D)(k-1-6) \leqslant-6 c$, and we obtain the result.

One can however get better upper bounds following the method used by Gallai $[13,14]$ for getting lower bounds on the density of critical graphs. This method is based on the concept of blocks. Recall that a graph $G$ is non-separable if it is connected and $G-v$ is connected for all $v \in V(G)$, and that a block of $G$ is a subgraph which is non-separable 
and maximal with respect to this property. Let $A$ be the set of cut-vertices of $G$ and $\mathcal{B}$ the set of blocks of $G$. The block forest $B(G)$ of $G$ is the graph on vertices $A \cup \mathcal{B}$ where $a B$ is an edge of $B(G)$ if and only if $a \in A, B \in \mathcal{B}$ and $a \in B$. The block forest of a graph is a forest. If $G$ is connected, then $B(G)$ is also connected. It is then called the block tree of $G$. A leaf block of a graph is a block which is a leaf in the block forest. Such a block has exactly one vertex in the union of all other blocks. This vertex is the attachment of the leaf block. The blocks and the block forest of a digraph are simply those of its underlying multigraph. A directed Gallai forest is a digraph in which each block is a single arc, a directed cycle, a bidirected odd cycle, or a bidirected clique.

Theorem 30 (Bang-Jensen et al.. [3]). Let $\vec{G}$ be a $k$-dicritical digraph. The subdigraph induced by the vertices of in- and out-degree $k-1$ is a directed Gallai forest.

Lemma 31. Let $k \geqslant 3$ be an integer. If $H$ is a directed Gallai forest of maximum degree at most $2 k$ not containing $\overleftrightarrow{K}_{k+1}$, then

$$
m(H) \leqslant\left(k-1+\frac{2}{k}\right) n(H) .
$$

Proof. We prove the result by induction.

If $H$ is not connected, then we have the result by applying the induction hypothesis on each of its connected components, and summing the obtained inequalities. If $H$ consists of a single block, then $H$ is either an arc, a directed cycle, a bidirected odd cycle, or a bidirected clique of order at most $k$. Hence $m(H) \leqslant(k-1) n(H)$, so we have the result.

Suppose now that $H$ is connected but not 2-connected.

Assume moreover that $H$ contains a leaf block $H_{1}$ which is not $\overleftrightarrow{K}_{k}$. Then

$$
m\left(H_{1}\right) \leqslant\left(k-1+\frac{2}{k}\right)\left(n\left(H_{1}\right)-1\right) .
$$

Let $H_{2}$ be the union of the blocks distinct from $H_{1}$. By the induction hypothesis, we have

$$
m\left(H_{2}\right) \leqslant\left(k-1+\frac{2}{k}\right) n\left(H_{2}\right)
$$

Because $n(H)=n\left(H_{1}\right)+n\left(H_{2}\right)-1$ and $m(H)=m\left(H_{1}\right)+m\left(H_{2}\right)$, we get the result by summing the two above inequalities.

Henceforth, assume that every leaf block is $\overleftrightarrow{K}_{k}$. Let $L$ be a leaf block of $H$ which is the end of a diameter $\mathbb{D}$ in the block tree. Let $P$ be the block incident to $L$ in $H$. It has maximum degree at most 2 and thus it is a directed cycle or a single arc. In particular, $P$ is not a leaf block, and so it is not an end of $\mathbb{D}$. Let $Q$ be the block distinct from $L$ which is incident to $P$ in $\mathbb{D}$, and let $L_{1}, \ldots, L_{q}$ be the blocks incident to $P$ and distinct from $Q$. Since $\mathbb{D}$ is a diameter, each $L_{i}$ is a leaf block and thus a $\overleftrightarrow{K}_{k}$. In particular, it implies that $q \leqslant n(P)-1$. Set $a=n(P)-q-1$, and note that $a \geqslant 0$. Let $H_{1}=P \cup \bigcup_{i=1}^{q} L_{i}$. We have $m\left(H_{1}\right)=q k(k-1)+n(P)=q k(k-1)+q+1+a$ and $n\left(H_{1}\right)=q(k-1)+n(P)=q k+1+a$. 


$$
\begin{aligned}
m\left(H_{1}\right) & =q k(k-1)+q+1+a \\
& \left.\leqslant q k(k-1)+2 q+\left(k-1+\frac{2}{k}\right) a \quad \text { (because } q \geqslant 1 \text { and } a \geqslant 0\right) \\
& \leqslant\left(k-1+\frac{2}{k}\right) q k+\left(k-1+\frac{2}{k}\right) a=\left(k-1+\frac{2}{k}\right)\left(n\left(H_{1}\right)-1\right)
\end{aligned}
$$

Let $H_{2}$ be the union of the blocks which do not appear in $H_{1}$. By the induction hypothesis, we have

$$
m\left(H_{2}\right) \leqslant\left(k-1+\frac{2}{k}\right) n\left(H_{2}\right) .
$$

Because $n(H)=n\left(H_{1}\right)+n\left(H_{2}\right)-1$ and $m(H)=m\left(H_{1}\right)+m\left(H_{2}\right)$, we get the result by summing the two above inequalities.

Theorem 32. Let $k \geqslant 3$ be an integer. Let $D$ be $a(k+1)$-dicritical digraph different from $\overleftrightarrow{K}_{k+1}$. Then

$$
m(D) \geqslant\left(k+\frac{k-2}{2 k^{2}+3 k-4}\right) n(D) .
$$

Proof. Let $S$ be the set of vertices $v \in V(D)$ such that $d^{+}(v)=d^{-}(v)=k$. By Theorem 30, the induced subdigraph $D\langle S\rangle$ is a directed Gallai forest, and by Lemma 31,

$$
m(D\langle S\rangle) \leqslant\left(k-1+\frac{2}{k}\right)|S| .
$$

Note that $2 k|S|$ is the number of arcs of $D$ incident with vertices of $\mathrm{S}$, counting those in $D\langle S\rangle$ twice. Hence,

$$
m(D) \geqslant 2 k|S|-m(D\langle S\rangle) \geqslant\left(k+1-\frac{2}{k}\right)|S| .
$$

All vertices in $V(D) \backslash S$ have degree at least $2 k+1$, so

$$
2 m(D) \geqslant(2 k+1)(n(D)-|S|)+2 k|S|=(2 k+1) n(D)-|S| .
$$

Considering $\left(k+1-\frac{2}{k}\right)$ Eq.(10) + Eq.(9), we obtain

$$
\begin{aligned}
\left(2 k+3-\frac{4}{k}\right) m(D) & \geqslant(2 k+1)\left(k+1-\frac{2}{k}\right) n(D) \\
\frac{2 k^{2}+3 k-4}{k} m(D) & \geqslant \frac{2 k^{3}+3 k^{2}-3 k-2}{k} n(D) \\
m(D) & \geqslant \frac{2 k^{3}+3 k^{2}-3 k-2}{2 k^{2}+3 k-4} n(D)=\left(k+\frac{k-2}{2 k^{2}+3 k-4}\right) n(D) .
\end{aligned}
$$


Corollary 33. For any surface $\Sigma$ and any $k \geqslant 6$, there is a finite number of $(k+1)$ dicritical digraphs embeddable on $\Sigma$.

Proof. Let $\Sigma$ be a surface, and $k \geqslant 6$. Set $\epsilon_{k+1}=\frac{k-2}{2 k^{2}+3 k-4}$ and $c=c(\Sigma)$. Let $D$ be a $(k+1)$-dicritical digraph embeddable on $\Sigma$ distinct from $\overleftrightarrow{K}_{k+1}$. Since there are at most two arcs between any vertices, by Theorem 2 , we have $m(D) \leqslant 6 n(D)-6 c$. Moreover, by Theorem $32, m(D) \geqslant\left(k+\epsilon_{k+1}\right) n(D)$. Hence $\left(k+\epsilon_{k+1}\right) n(D) \leqslant 6 n(D)-6 c$ so

$$
n(D) \leqslant \frac{-6 c}{k-6+\epsilon_{k+1}} .
$$

\subsection{Dicolouring planar digraphs and planar oriented graphs}

Theorem 34. Deciding whether a planar digraph is 2-dicolourable is NP-complete.

Proof. We shall give a reduction from PLANAR 3-SAT which consists in deciding whether a 3-SAT formula whose incidence graph ${ }^{1}$ is planar is satisfiable. This problem was shown to be NP-complete by Lichtenstein [26].

A $\neq$-gadget between $u$ and $v$ is a digon between $u$ and $v$. Trivially, a $\neq$-gadget is 2-dicolourable and its extremities have distinct colours in any of its 2-dicolourings.

Consider now an instance $\Phi$ of PLANAR 3-SAT and let $H$ be its incidence graph embedded in the plane. Let us construct the planar oriented graph $\vec{G}$ from $H$ as follows. First, we add a vertex $t_{F}$ in each face of $H$. Now for every clause $C=\ell_{x} \vee \ell_{y} \vee \ell_{z}$, we replace the vertex $C$ and the three incident edges by a clause gadget as follows. We replaced the vertex $C$ by a directed 6 -cycle $\left(x_{C}, u_{C}, y_{C}, v_{C}, z_{C}, w_{C}, x_{C}\right)$ inside which we add a vertex $t_{C}$ which is connected to $u_{C}, v_{C}$ and $w_{C}$ via $\neq$-gadgets. Let $F_{1}$ (resp. $F_{2}, F_{3}$ ) be the face containing $(x, C, y)(\operatorname{resp}(y, C, z),(z, C, x))$ in $H$. We add a $\neq$-gadget between $t_{F_{1}}$ and $u_{C}$, between $t_{F_{2}}$ and $v_{C}$, and between $t_{F_{3}}$ and $w_{C}$. Finally, for any $a \in\{x, y, z\}$, if $\ell_{a}$ is the negated literal $\bar{a}$, then add a $\neq$-gadget between the variable vertex $a$ and $a_{C}$, and if $\ell_{a}$ is the non-negated literal $a$, the add a new vertex $\bar{a}_{C}$ and two $\neq$-gadgets between this vertex and $a$ and $a_{C}$. See Figure 3 .

Let us now show that $\Phi$ is satisfiable if and only if $\vec{G}$ is 2-dicolourable.

Assume first that $\Phi$ is satisfiable. Colour each variable with 1 if it is true and 2 if it is false. For each face $F$ of $H$, colour the vertex $t_{F}$ with 1 , and for each clause $C$ colour the vertex $t_{C}$ with 1 and $u_{C}, v_{C}, w_{C}$ with 2 . It remains to colour $x_{C}, y_{C}$ and $z_{C}$ for each clause $C$. Each of these vertices is incident with a unique $\neq$-gadget, which forces to colour the vertex with the colour opposite to the one of the other end of the gadget. Let us show that there is no monochromatic directed cycle. Assume for a contradiction that there is such a cycle. Since two vertices linked by a $\neq$-gadget have distinct colours, such a cycle can only be one of the cycles $\left(x_{C}, u_{C}, y_{C}, v_{C}, z_{C}, w_{C}, x_{C}\right)$ for some clause $C=\ell_{x} \vee \ell_{y} \vee \ell_{z}$. But, as $\Phi$ is satisfied, at least one of the literals $\ell_{x}, \ell_{y}, \ell_{z}$ is true, and thus, by construction, at least

\footnotetext{
${ }^{1}$ The incidence graph of a 3-SAT formula is the bipartite graph with a vertex for each clause and each variable, and a variable is adjacent to a clause if it belongs to it.
} 


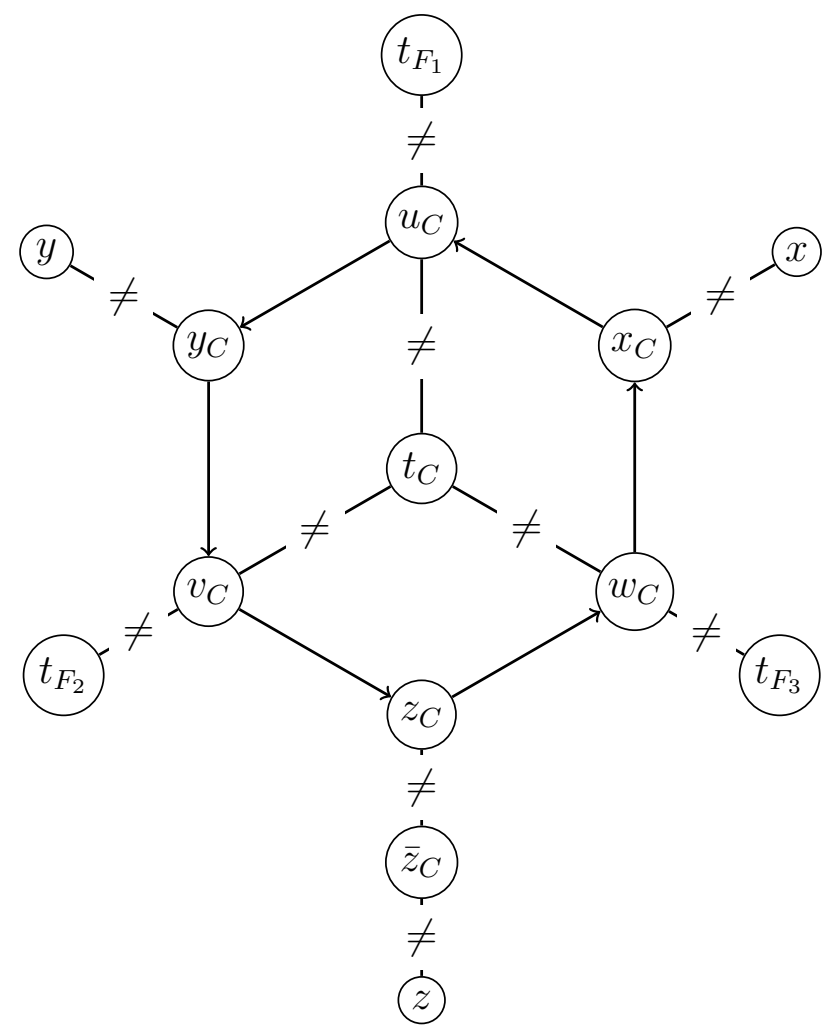

Figure 3: Clause gadget associated to the clause $\neg x \vee \neg y \vee z$.

one of the vertices $x_{C}, y_{C}, z_{C}$ is coloured 1 . But the vertices $u_{C}, v_{C}, w_{C}$ are coloured 2 , so the cycle is not monochromatic. Hence we have a 2-dicolouring of $\vec{G}$.

Assume now that $\vec{G}$ admits a 2-dicolouring. Observe that the digraph induced by the $t_{F}$ for $F$ face of $H$, and all the $t_{C}, u_{C}, v_{C}, w_{C}$ for $C$ clause, and all the $\neq$-gadgets between them, is connected. Without loss of generality, we may assume that all the $t_{F}$ and $t_{C}$ are coloured 1 and all the $t_{C}, u_{C}, v_{C}, w_{C}$ are coloured 2 . Let $\phi$ be the truth assignment defined by $\phi(x)=$ true if and only if $x$ is coloured $1 \mathrm{in} \vec{G}$. Consider a clause $C=\ell_{x} \vee \ell_{y} \vee \ell_{z}$. The directed cycle $\left(x_{C}, u_{C}, y_{C}, v_{C}, z_{C}, w_{C}, x_{C}\right)$ is not monochromatic, so at least one vertex $x_{C}$, $y_{C}$ and $z_{C}$ is coloured 1. By construction, this means that one of the literals $\ell_{x}, \ell_{y}, \ell_{z}$ is true. Hence $\Phi$ is satisfied.

Since every graph embeddable on the sphere can also be embedded in any other surface, Theorem 34 implies that $\Sigma$-2-DicolourABILITY is NP-complete for any surface $\Sigma$. It is then natural to ask about the complexity of the problem restricted to oriented graphs.

Recall that Conjecture 7 states that every planar oriented graph is 2-dicolourable. If true, it implies that $\mathbb{S}_{0}$-OrIEnTED-2-Dicolourability can be trivially solved in polynomial time because the answer is always positive. The following result shows that, conversely, if it happens to be false, $\mathbb{S}_{0}$-ORIEnTED-2-DiCOlourABILITY is NP-complete.

Theorem 35. If Conjecture 7 does not hold, then deciding whether a planar oriented graph is 2-dicolourable is NP-complete. 
Proof. The proof is similar to the one of Theorem 34. The only difference is in the $\neq$-gadget, which should now be constructed without any digons.

Suppose that Conjecture 7 does not hold. Then there is a planar 3-dicritical oriented graph $\vec{G}$. Let $u v$ be an arc of $\vec{G}$. By definition of 3-dicriticality, $\vec{G} \backslash u v$ is 2-dicolourable. Moreover, in any 2-dicolouring $\phi$ of $\vec{G} \backslash u v, u$ and $v$ are coloured the same for otherwise $\phi$ would be a 2-dicolouring of $\vec{G}$. We say that $\vec{G} \backslash u v$ is a =-gadget between $u$ and $v$.

Let us now explain how to construct a $\neq$-gadget between two vertices $u$ and $w$. We start with four vertices $u, v_{1}, v_{2}, w$. We add the $\operatorname{arcs} v_{1} v_{2}, v_{2} w, w v_{1}$ and two =-gadgets between $u$ and $v_{1}$ and between $u$ and $v_{2}$. See Figure 4 . One easily sees that a $\neq$-gadget is 2-dicolourable and that $u$ and $w$ have distinct colours in any of its 2-dicolourings.

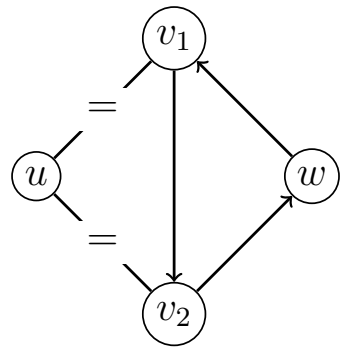

Figure 4: A $\neq$-gadget between $u$ and $w$.

\section{Concluding remarks}

We have determined the dichromatic number of the projective plane $\mathbb{N}_{1}$, the Klein bottle $\mathbb{N}_{2}$, the torus $\mathbb{S}_{1}$, Dyck's surface $\mathbb{N}_{3}$, the 5 -torus $\mathbb{S}_{5}$, and the 10 -cross surface $\mathbb{N}_{10}$. For the surfaces in between, the dichromatic number is either 3 or 4 . We verified that all orientations of triangulations of the double torus $\mathbb{S}_{2}$ or $\mathbb{N}_{4}$ on at most 14 vertices have dichromatic number at most 3 by computer. Note that this does not imply that all 14-vertex graphs embeddable on these surfaces have dichromatic number at most 3, see e.g. [7]. By Theorem 10, it would suffice to check all digraphs on up to 22 vertices.

Problem 36. Determine the maximum dichromatic number of an oriented graph embeddable on the double torus $\mathbb{S}_{2}$ or in the 4-cross surface $\mathbb{N}_{4}$.

A common generalization of colouring is list colouring. Similarly, dicolouring generalizes to list dicolouring. A digraph $D$ is $\boldsymbol{k}$-list-dicolourable if it is $L$-dicolourable for every list assignment $L$ such that $|L(v)| \geqslant k$ for all $v \in V(D)$. The list dichromatic number of a digraph $D$ is the least integer $k$ such that $D$ is $k$-list-dicolourable. Note that by degeneracy, every planar oriented graph is 3-list-dicolourable. It has been asked whether the list version of Conjecture 7 holds, see [4]. Combining degeneracy and Theorem 26, one can show that oriented graphs on the projective plane, the Klein bottle and the torus are 3-list-dicolourable. However, our proofs of Theorems 23 and 27 do not generalize. 
Problem 37. Determine the maximum list dichromatic number of an oriented graph embeddable in Dyck's surface $\mathbb{N}_{3}$.

We further believe that the asymptotic behaviour of the list dichromatic number of surfaces is an interesting topic of future research.

Recall that Conjecture 7 holds for digraphs of digirth 4. Indeed, the proof of [25] gives a decomposition of planar triangulations into two induced chordal graphs. Any such decomposition of a graph is a 2-dicolouring for all its orientations of digirth 4. Unfortunately, there are graphs embeddable in the projective plane that cannot be decomposed into two induced chordal graphs, see the left of Figure 5. On the other hand, we have verified that all orientations of triangulations in $\mathbb{N}_{1}$ with digirth 4 and at most 17 vertices have dichromatic number 2 . Indeed, the smallest 3-dichromatic oriented graph of digirth 4 that we know of contains a $K_{5,8}$, see the right of Figure 5 . Hence, it is not embeddable in $\mathbb{N}_{10}$ nor $\mathbb{S}_{5}$, see $[6]$.

Problem 38. Determine the maximum dichromatic number of an oriented graph with digirth 4 embeddable on the projective plane.
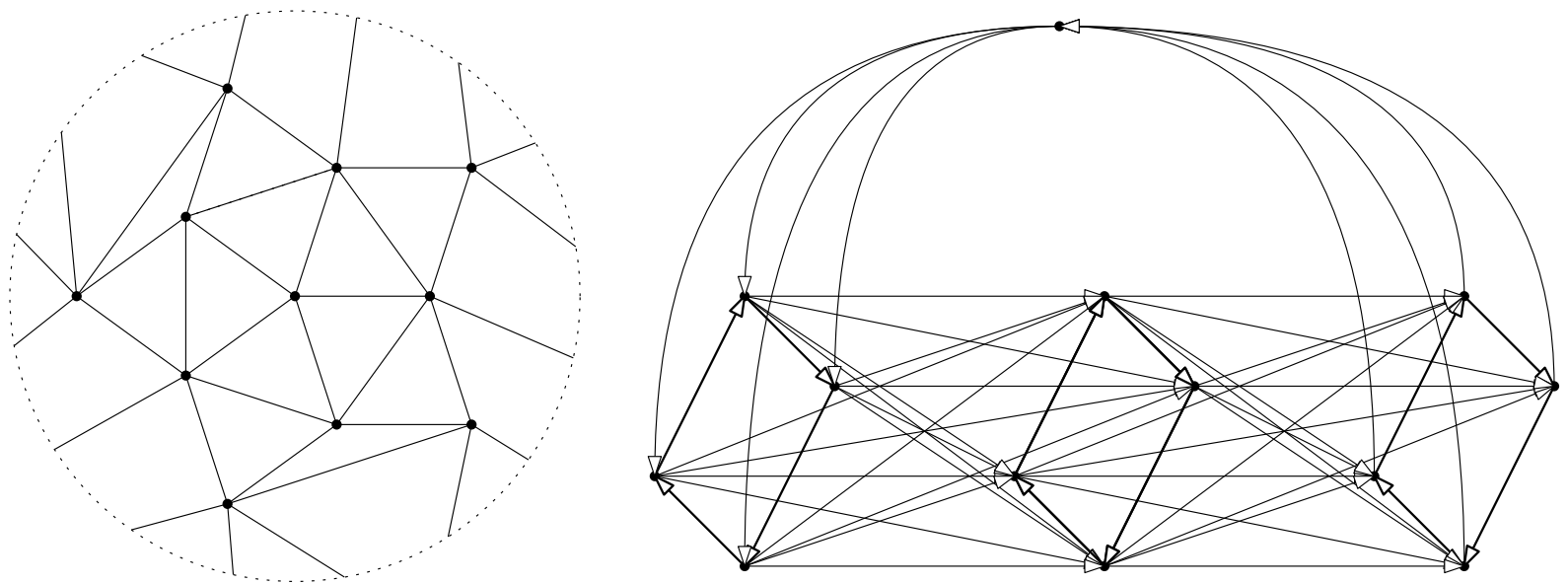

Figure 5: Left: A triangulation of $\mathbb{N}_{1}$ that cannot be decomposed into two chordal graphs. Right: A 3-dichromatic digraph of digirth 4.

On the other hand it follows from Theorem 10, that for a digraph $D$ on $\Sigma$ of Euler characteristic $c$, one has that digirth more than $4-9 c$ implies $\chi(\vec{D}) \leqslant 3$. We believe that the interplay of digirth, genus, and dichromatic number deserves further investigation.

We prove that the number of $k$-dicritical digraphs embeddable on a surface is finite for any $k \geqslant 7$. Thus, for $k \geqslant 6 \Sigma$ - $k$-Dicolourability is polynomial time solvable. On the other hand we show that $\Sigma$-2-DicolourABILITY is NP-complete. Since $\chi(G)=\vec{\chi}(\overleftrightarrow{G})$, the NP-completeness of the 3-colourability of graph embedded in any fixed surface implies that $\Sigma$-3-Dicolourability is NP-complete for all surface $\Sigma$. The following remains. 
Problem 39. Let $\Sigma$ be a surface different from the sphere and $k \in\{4,5\}$. What is the complexity of $\Sigma$ - $k$-DiCOLOURABILITY ? Are there a infinitely many 6 -dicritical digraphs embeddable on $\Sigma$ ?

We further show that if Conjecture 7 is false, then $\mathbb{S}_{0}$-OrIEnTED-2-DicolourABILITY is NP-complete. However, the method of this proof does not extend to prove the NP-completeness of $\Sigma$-ORIENTED-2-DicolourABILITy for a surface $\Sigma$ other than the sphere. Indeed, while assembling together planar gadgets results in a planar graph, assembling gadgets embeddable on a given surface does not necessarily results in a graph embeddable on this surface.

Problem 40. Let $\Sigma$ be a surface. What is the complexity of $\Sigma$-ORIEnTED-2DiCOLOURABILITY?

\section{Acknowledgements}

We thank Stéphane Bessy, Louis Esperet, Ararat Harutyunyan, Jocelyn Thiebaut, and Petru Valicov for fruitful discussions. We particularly thank Raphael Steiner for pointing us to the argument for the upper bound in Theorem 21. This research was supported by projects Digraphs: ANR-19-CE48-0013-01, GATO: ANR-16-CE40-0009-01, ALCOIN: PID2019-104844GB-I00, RYC-2017-22701, ALGORIDAM: ANR-19-CE48-0016 and ANR project DAGDigDec (JCJC) ANR-21-CE48-0012. An extended abstract of this paper has been published at EuroComb 2021 [1].

\section{References}

[1] P. Aboulker, F. Havet, K. Knauer, C. Rambaud. On the Dichromatic Number of Surfaces. In: Nešetřil J., Perarnau G., Rué J., Serra O. (eds) Extended Abstracts EuroComb 2021. Trends in Mathematics, vol 14. Birkhäuser, Cham. 2021.

[2] K. Appel and W. Haken. Every planar map is four colorable. Part I: Discharging. Illinois J. Math., 21(3):429-490, 091977.

[3] J. Bang-Jensen, T. Bellitto, M. Stiebitz, and T. Schweser. Hajós and ore constructions for digraphs Electron. J. Comb., 27(1), \#P1.63, (2020). 2019.

[4] J. Bensmail, A. Harutyunyan, and N. K. Le. List coloring digraphs. J. Graph Theory, 87(4):492-508, 2018.

[5] D. Bokal, G. Fijavz, M. Juvan, P. M. Kayll, and B. Mohar. The circular chromatic number of a digraph. J. Graph Theory, 46(3):227-240, 2004.

[6] A. Bouchet. Orientable and nonorientable genus of the complete bipartite graph. J. Comb. Theory, Series B, 24(1):24-33, 1978.

[7] J. Davies and F. Pfender. Edge-maximal graphs on orientable and some nonorientable surfaces, J. Graph Theory 98(3):405-425, 2021. 
[8] G. A. Dirac. Map colour theorems related to the Heawood colour formula. II. J. London Math. Soc., 32:436-455, 1957.

[9] P. Erdős, J. Gimbel, and D. Kratsch. Some extremal results in cochromatic and dichromatic theory. J. Graph Theory, 15(6):579-585, 1991.

[10] P. Erdős and L. Moser. On the representation of directed graphs as unions of orderings. Magyar Tud. Akad. Mat. Kutató Int. Közl., 9:125-132, 1964.

[11] S. Fisk. The nonexistence of colorings. J. Comb. Theory, Series B, 24(2):247-248, 1978.

[12] P. Franklin. A six-color problem. J. Math. Phys., 13:363-369, 1934.

[13] T. Gallai. Kritische Graphen. I. Magyar Tud. Akad. Mat. Kutató Int. Közl., 8:165$192,1963$.

[14] T. Gallai. Kritische Graphen. II. Magyar Tud. Akad. Mat. Kutató Int. Közl., 8:373395 (1964), 1963.

[15] M. R. Garey and D. S. Johnson. Computers and intractability. W. H. Freeman and Co., San Francisco, Calif., 1979. A guide to the theory of NP-completeness, A Series of Books in the Mathematical Sciences.

[16] J. Gimbel and C. Thomassen. Coloring graphs with fixed genus and girth. Trans. Am. Math. Soc, 349(11):4555-4564, 1997.

[17] H. Grötzsch. Zur Theorie der diskreten Gebilde. VII. Ein Dreifarbensatz für dreikreisfreie Netze auf der Kugel. Wiss. Z. Martin-Luther-Univ. Halle-Wittenberg. Math.Nat. Reihe, 8:109-120, 1958/1959.

[18] A. Harutyunyan and B. Mohar. Gallai's theorem for list coloring of digraphs. SIAM J. Discrete Math., 25:170-180, 012011.

[19] P. J. Heawood. Map colour theorems. Quart. J. Pure Appl. Math., 24:332-338, 1890.

[20] K. Knauer and P. Valicov. Cuts in matchings of 3-connected cubic graphs. Eur. J. Comb., 76:27-36, 2019.

[21] A. V. Kostochka and M. Stiebitz. The minimum number of edges in 4-critical digraphs of given order. Graphs Comb., 36(3):703-718, 2020.

[22] H. V. Kronk. An analogue to the Heawood map-colouring problem. J. London Math. Soc., s2-1(1):750-752, 1969.

[23] H. V. Kronk. The chromatic number of triangle-free graphs. In Y. Alavi, D. R. Lick, and A. T. White, editors, Graph Theory and Applications, pages 179-181, Berlin, Heidelberg, 1972. Springer Berlin Heidelberg.

[24] H. V. Kronk and A. T. White. A 4-color theorem for toroidal graphs. Proc. Natl. Acad. Sci. U.S.A., 34(1):83-86, 1972.

[25] Z. Li and B. Mohar. Planar digraphs of digirth four are 2-colorable. SIAM J. Discrete Math., 31(3):2201-2205, 2017.

[26] D. Lichtenstein. Planar formulae and their uses. SIAM J. Comput., 11(2):329-343, 1982. 
[27] B. D. McKay and A. Piperno. Practical graph isomorphism, \{II $\}$. J. Symb. Comput., 60(0):94-112, 2014.

[28] V. Neumann-Lara. The dichromatic number of a digraph. J. Comb. Theory, Series B, 33(3):265-270, 1982.

[29] V. Neumann-Lara. The 3 and 4-dichromatic tournaments of minimum order. Discret. Math., 135(1-3):233-243, 1994.

[30] K. Reid and E. Parker. Disproof of a conjecture of Erdos and Moser on tournaments. J. Comb. Theory, 9(3):225-238, 1970.

[31] G. Ringel and J. W. T. Youngs. Solution of the Heawood map-coloring problem. Proc. Natl. Acad. Sci. U.S.A., 60(2):438-445, 1968.

[32] N. Robertson, D. P. Sanders, P. D. Seymour, and R. Thomas. A new proof of the fourcolour theorem. Electron. Res. Announc. Amer. Math. Soc., 2(1):17-25 (electronic), 1996.

[33] A. Sanchez-Flores. On tournaments free of large transitive subtournaments. Graphs Combin., 14(2):181-200, 1998.

[34] R. Stearns. The voting problem. Am. Math. Mon., 66:761-763, 1959.

[35] C. Thomassen. Grötzsch's 3-color theorem and its counterparts for the torus and the projective plane. J. Comb. Theory, Series B, 62(2):268-279, 1994.

[36] C. Thomassen. Color-critical graphs on a fixed surface. J. Comb. Theory, Series B, 70(1):67-100, 1997. 